\title{
Templated Synthesis of Polymer-Based Yolk/Shell Particles with Tunable Morphologies
}

Xuanrong Guo, ${ }^{1}$ Kiara Santiago González, ${ }^{1}$ and David M. Lynn ${ }^{1,2, *}$

${ }^{1}$ Department of Chemical and Biological Engineering, 1415 Engineering Drive, and ${ }^{2}$ Department of Chemistry, 1101 University Avenue, University of Wisconsin - Madison, Madison, Wisconsin 53706.

\section{Supporting Information}
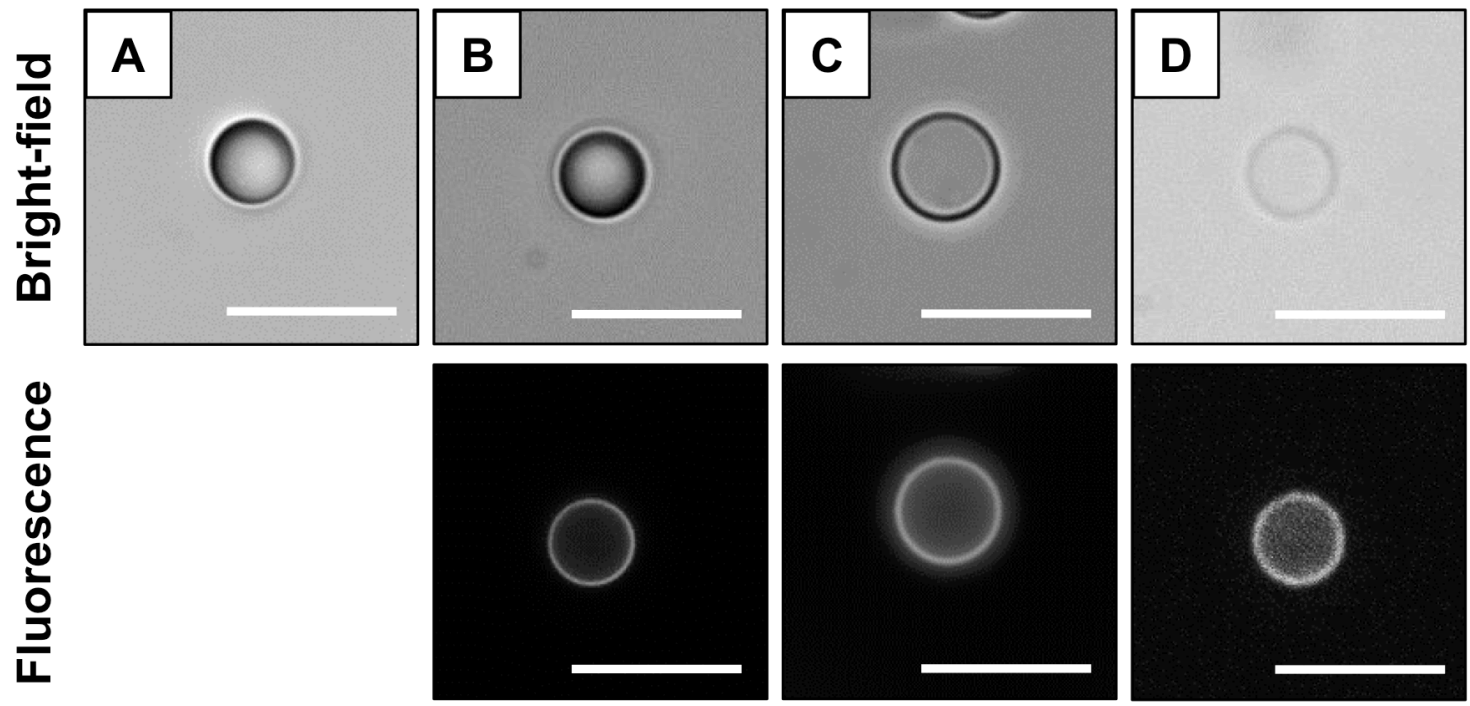

Figure S1: Bright-field (top row) and fluorescence (bottom row) microscopy images showing (A) a bare silica particle template in water, (B) a multilayer PVDMA/Cys film-coated silica particle in water, (C) a PVDMA/Cys capsule in tetradecane, and (D) a PVDMA/Cys capsule in polymerizable mixtures of BzMA and HDODA monomers (see the main text). Scale bars are $10 \mu \mathrm{m}$.
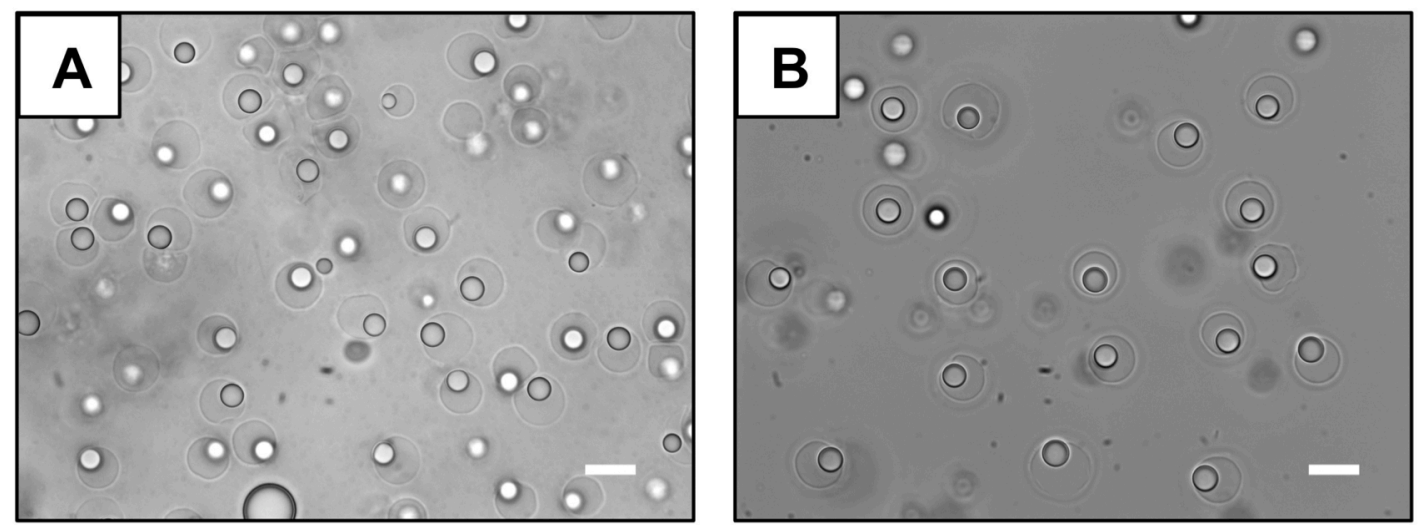

Figure S2: Bright-field images showing (A) PVDMA/Cys capsules filled with BzMA and HDODA monomers that are not labeled with fluorescent dyes (see the main text) suspended in deionized water and (B) the corresponding polymer yolk/shell particles after UV illumination. Scale bars are $10 \mu \mathrm{m}$. 


\section{Captions to Accompany Videos S1-S4:}

Video S1: Video acquired using bright-field light microscopy showing the mobilities of encapsulated reactive oil droplets before and after polymerization in DI water. Free-floating yolk/shell-type capsules were electrostatically immobilized on a planar surface prior to imaging (see main text). Approximately $20 \%$ of the encapsulated liquid oil droplets or the solid polymer yolks were observed to rotate and translate within the confines of the surrounding polymer cages. The video shown here has been adjusted to run at $4 \mathrm{X}$ the original speed and shows video acquired in the following sequence: (i) bright-field before polymerization and (ii) bright-field after polymerization in DI water. See main text for additional details of these experiments.

Video S2: Video acquired using bright-field light microscopy showing the mobilities of encapsulated reactive oil droplets before and after polymerization in the presence of $1 \mathrm{mM}$ DTAB. Free-floating yolk/shell-type capsules were electrostatically immobilized on a planar surface prior to imaging (see main text). Approximately $90 \%$ of the encapsulated liquid oil droplets or the solid polymer yolks were observed to rotate and translate within the confines of the surrounding polymer cages. The video shown here has been adjusted to run at $4 \mathrm{X}$ the original speed and shows video acquired in the following sequence: (i) bright-field before polymerization and (ii) bright-field after polymerization in the presence of $1 \mathrm{mM}$ DTAB. See main text for additional details of these experiments.

Video S3: Video acquired using bright-field light microscopy showing the mobilities of encapsulated reactive oil droplets before and after polymerization in the presence of $200 \mu \mathrm{M}$ SDS. Free-floating yolk/shell-type capsules were electrostatically immobilized on a planar surface prior to imaging (see main text). Most of the encapsulated liquid oil droplets or the solid polymer yolks were immobile. The video shown here has been adjusted to run at $4 \mathrm{X}$ the original speed and shows video acquired in the following sequence: (i) bright-field before polymerization and (ii) bright-field after polymerization in the presence of $200 \mu \mathrm{M}$ SDS. See main text for additional details of these experiments.

Video S4: Video acquired using bright-field light microscopy showing decreases in the sizes of the encapsulated monomer droplets after aging the aqueous suspension of monomer-filled capsules with an oil phase (see main text and Figure 7). As the droplets become smaller, some of the droplets that wet the inner surfaces of the surrounding polymer capsules and are immobile in their initial states are observed to detach from the surface of the capsule wall, spontaneously forming free-floating encapsulated droplets in the absence of added DTAB (marked by asterisks; see Figure 7). The video shown here has been adjusted to run at $7200 X$ the original speed. See main text for additional details of these experiments. 